\title{
DIVERSIDADE EM QUATRO ÁREAS de CAMPOS RUPESTRES NA CHAPADA Diamantina, BaHIA, BRASIL: ESPÉCIES DISTINTAS, MAS RIQUEZAS SIMILARES ${ }^{1}$
}

\author{
Abel Augusto Conceição ${ }^{1} \&$ José Rubens Pirani ${ }^{2}$
}

\begin{abstract}
Resumo
(Diversidade em quatro áreas de campos rupestres na Chapada Diamantina, Bahia, Brasil: espécies distintas, mas riquezas similares) O presente estudo teve como objetivo revelar padrões de diversidade nos campos rupestres, gerando informações úteis à conservação. O estudo desenvolvido em quatro locais na Chapada Diamantina, todos eles em topos de serras e morros, incluiu dois tipos principais de fisionomias abertas: uma mais campestre e contínua (habitat entremeio) e outra mais arbustiva e insular (habitat afloramento). A vegetação foi amostrada em cada um dos quatro locais por quatro parcelas aleatórias simples de $10 \times 10 \mathrm{~m}$. Além dessas 16 parcelas, mais 16 foram estratificadas ao habitat afloramento, quatro em cada local. Foram analisadas as riquezas em espécies, famílias, criptógamas vasculares, eudicotiledôneas, monocotiledôneas, além das estimativas das áreas de coberturas das plantas vasculares (vegetação) e dos liquens. Do total de 202 espécies, 11 foram criptógamas vasculares e 191 foram angiospermas ( 97 monocotiledôneas, 93 eudicotiledôneas e uma Piperaceae). Foi verificado aumento do número de espécies conforme os aumentos de área de vegetação e de inclinação até $13^{\circ}$. A variação da área de vegetação entre os locais não foi significativa, assim como as riquezas em espécies, em famílias, em espécies de eudicotiledôneas e monocotiledôneas, sugerindo a existência de certa estabilidade, apesar do dendrograma de similaridade demonstrar que a composição de espécies entre os locais varia. Diferenças significativas entre as áreas de liquens e o número de espécies criptógamas vasculares indicam algumas distinções entre os locais, provavelmente decorrentes de fatores como umidade e isolamento.
\end{abstract}

Palabras chave: campo rupestre, Cadeia do Espinhaço, florística, riqueza, vegetação em montanha.

\begin{abstract}
(Diversity of four sites on 'campos rupestres' in the Chapada Diamantina, Bahia, Brasil: different compositions but similar richness) This study aims at detecting patterns of diversity on 'campos rupestres', providing useful information to conservation strategies. Four sites in the Chapada Diamantina's summits were studied, including two kinds of open physiognomies, one more grassy and homogenous (continuous habitat) and the other more shrubby and heterogeneous (insular habitat). The vegetation in each one of the four sites was sampled by four simple random $10 \times 10 \mathrm{~m}$ units. Besides these 16 units, more 16 were stratified to the insular habitat, four in each site. The number of species, families, vascular cryptogams, eudicots, and monocots, besides coverage areas estimations of vascular plants (vegetation) and lichens were analyzed. The total number of species was 202, 11 vascular cryptogams and 191 flowering plants (97 monocots, 93 eudicots, and one Piperaceae). The number of species increases with vegetation area and slope up to $13^{\circ}$. Similarity cluster shows that species compositions are different among the sites studied. Nevertheless, vegetation area between them did not show significant differences, like species richness, family richness, mono and eudicot richness, suggesting a relative steady system. The areas of lichens and vascular cryptogams were different among sites, which is probably affected by different humidity and insulation.
\end{abstract}

Key words: campo rupestre, Espinhaço Range, floristic, richness, mountain vegetation.

\footnotetext{
Artigo recebido em 07/2006. Aceito para publicação em 12/2006.

${ }^{1}$ Parte da Tese de Doutorado do primeiro autor. Financiamento FAPESP 99/05322-7

${ }^{1}$ Universidade Estadual de Feira de Santana, Departamento de Ciências Biológicas, km 03, BR 116, 44031-460, Feira de Santana, BA, Brasil. abel18@gmail.com

${ }^{2}$ Universidade de São Paulo, Instituto de Biociências, Departamento de Botânica, Cx. Postal 11461, 05422-970, São Paulo, SP, Brasil.
} 


\section{INTRODUÇÃO}

Os campos rupestres caracterizam-se pela vegetação predominantemente herbácea e arbustiva associada aos afloramentos rochosos e solos arenosos localizados nas áreas mais elevadas de serras, principalmente nas da Cadeia do Espinhaço. Muitas espécies exuberantes de famílias como Orchidaceae, Bromeliaceae, Eriocaulaceae, Cactaceae, Melastomataceae e Asteraceae são endêmicas dos campos rupestres, integrando uma paisagem única e dinâmica, com intensas floradas coloridas espalhadas pelas serras (Giulietti et al. 1987, 1997; Harley 1995; Stannard 1995; Zappi et al. 2003; Conceição et al. 2005).

Devido à ocorrência de mais de mil espécies endêmicas, o campo rupestre foi considerado uma "fitocória", que pela configuração insular (restrita aos topos de serras disjuntas) foi referida como "arquipélago" (Prance 1994). As serras constituem-se em sistemas isolados por terrenos mais baixos que atuariam como barreiras geográficas, interferindo na formação de floras autóctones (Giulietti \& Pirani 1988).

Estudos quantitativos da vegetação de campo rupestre vêm revelando elevada diversidade, tanto pelo número total de espécies, como para o das endêmicas e presença de habitats diferenciados, porém geralmente são restritos a um ou a dois locais (Andrade et al. 1986; Alves \& Kolbek 1993; Meguro et al. 1994; Pereira 1994; Pirani et al. 1994; Vitta 1995; Conceição \& Giulietti 2002; Conceição \& Pirani 2005).

Áreas distintas provêm informações importantes para compreensão dos padrões de diversidade (Rosenzweig 1995; Whittaker et al. 2001), especialmente no campo rupestre, já que este apresenta grande variação de composição entre áreas disjuntas (Zappi et al. 2003; Conceição \& Pirani 2005; Conceição et al. 2005). Grande parte dos estudos da vegetação nos campos rupestres refere-se aos levantamentos florísticos (Giulietti et al. 1987; Harley \& Simmons 1986; Stannard 1995; Guedes \& Orge 1998; Zappi et al. 2003), restando poucos que abordaram a diversidade de espécies com unidades amostrais de tamanho padronizado, como os realizados em porções da Cadeia do Espinhaço em Minas Gerais (Andrade et al. 1986; Pereira 1994; Vitta 1995) e na Bahia (Conceição \& Giulietti 2002; Conceição \& Pirani 2005). Comparações confiáveis das diversidades entre locais distintos precisam ser fundamentadas em estudos com delineamentos similares, de maneira que não interferiram nos padrões detectados (Whittaker et al. 2001; Pillar 2004).

Uma das características de sistemas ecológicos é sua variação não-aleatória, o que se manifesta na existência de padrões no espaço e no tempo, dependentes do tamanho das unidades amostrais (Pillar 2004), comprometendo comparações entre estudos dos campos rupestres que utilizaram tamanhos distintos de unidades. Os campos rupestres incluem uma mistura de habitats discernidos pela configuração do substrato, continuidade da vegetação, composição florística, proporção de rocha exposta, presença de blocos de rocha e de sedimentos arenosos, secos ou periodicamente encharcados na estação chuvosa, constituindo um sistema bastante heterogêneo, com mudanças bruscas de comunidades em um curto espaço (Conceição 2000; Conceição \& Giulietti 2002; Conceição 2003; Conceição \& Pirani 2005).

Sabendo que a composição de espécies nos campos rupestres muda entre locais e habitats, mesmo que esses sejam muito próximos (Conceição \& Giulietti 2002; Conceição \& Pirani 2005; Conceição et al. 2005) e que dois dos habitats mais comuns estudados no Morro da Mãe Inácia e na Fumaça possuem padrões distintos quanto às abundâncias proporcionais das espécies (Conceição \& Pirani 2005), será que o número de espécies ou o de famílias em diferentes locais e incluindo diferentes habitats é distinto?

O presente estudo objetiva revelar padrões mais gerais da diversidade dos campos rupestres nas montanhas da Chapada Diamantina, gerando informações úteis à conservação. 


\section{Material e Métodos}

\section{Áreas de estudo}

Foram estudadas quatro áreas de campo rupestre que incluíam grande proporção de rocha exposta em topos de morros e serras da Serra do Sincorá, Chapada Diamantina, Bahia

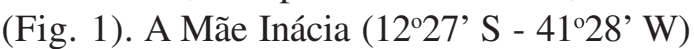
localiza-se no município de Palmeiras, sendo a única área fora do Parque Nacional da Chapada Diamantina, com altitudes entre 1100 a $1140 \mathrm{~m}$. Os afloramentos da Cachoeira da Fumaça (12 $\left.35^{\prime} \mathrm{S}-41^{\circ} 27^{\prime} \mathrm{W}\right)$ e os do Gerais da Fumaça (12³6' S - 41'28' W) situam-se na Serra da Larguinha, no município de Palmeiras, entre 1310 e $1360 \mathrm{~m}$ de altitude. Os afloramentos de Guiné (1245' S - 41 $\left.{ }^{\circ} 30^{\prime} \mathrm{W}\right)$, no município de Mucugê, situam-se na Serra do Esbarrancado. Eles são os mais altos, no limite oeste da Serra do Sincorá e do Parque Nacional da Chapada Diamantina, a cerca de $1400 \mathrm{~m}$ de altitude.

O clima nos locais estudados é do tipo Tropical do Brasil Central, subquente, semiúmido, com verão úmido e quatro a cinco meses secos concentrados na primavera (Nimer 1989). De 1998 a 2002, a precipitação anual nas proximidades dos locais estudados variou entre $837 \mathrm{~mm}$ em $1998 \mathrm{e} 1828 \mathrm{~mm}$ em 2002 (Conceição 2003). No período que se estende de junho a agosto os meses são mais frios e as médias de temperatura anuais nos locais com altitudes entre 1000 e 1100 m são inferiores a $20^{\circ} \mathrm{C}$, podendo ocorrer mínimas diárias inferiores a $4^{\circ} \mathrm{C}$ (Nimer 1989).

Arenitos e quartzitos formados na era PréCambriana compõem a topografia acidentada da Chapada Diamantina, decorrente da erosão diferencial (Moreira \& Camelier 1977). Os afloramentos rochosos estudados são incluídos no Grupo Chapada Diamantina, que se inicia próximo à Mucugê, estendendo-se até Santo Inácio, fazendo parte da Formação Tombador (Torquato \& Fogaça 1981). Em afloramentos rochosos nos topos de serras da Chapada Diamantina, os sedimentos onde a vegetação é associada às maiores proporções de rocha exposta são rasos, arenosos e ácidos, concentrando elevado teor de matéria orgânica e argilas em relação aos locais com vegetação mais contínua (Conceição \& Giulietti 2002; Conceição \& Pirani 2005).

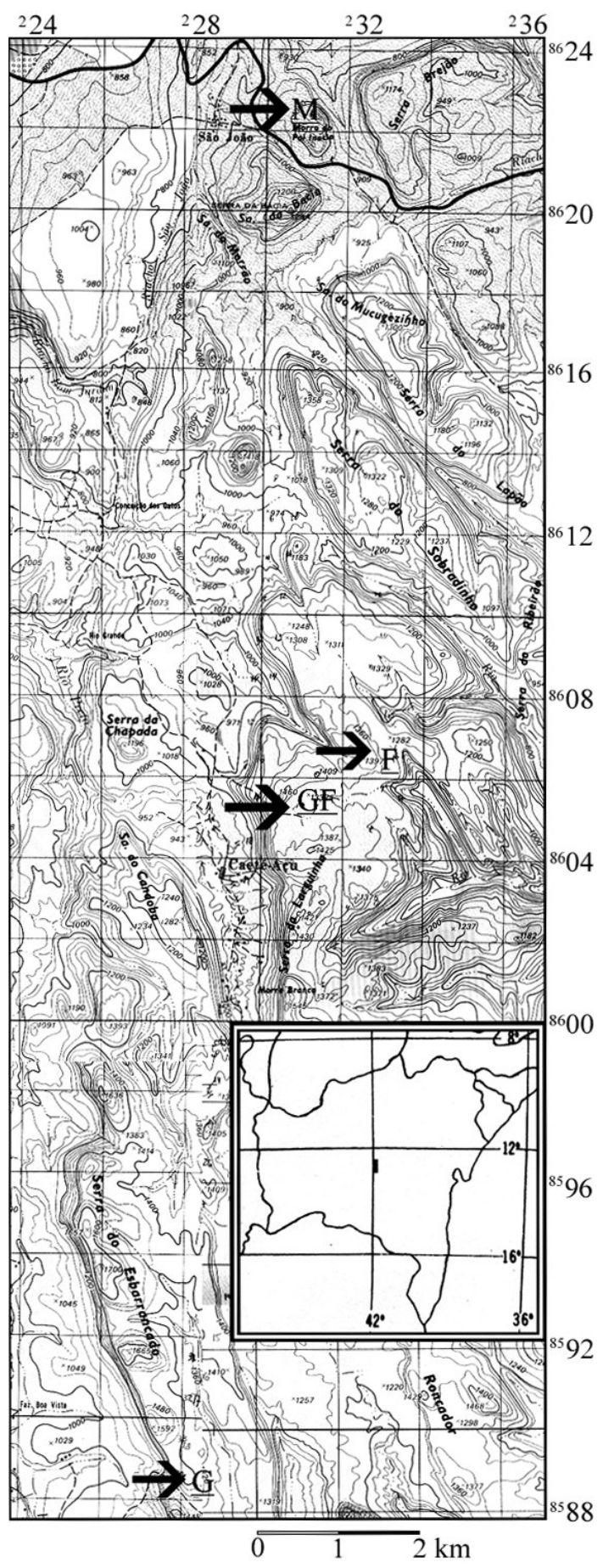

Figura 1 - Localização das quatro áreas estudadas na Chapada Diamantina, Bahia, Brasil. Mãe Inácia (M), Fumaça (F), Gerais da Fumaça (GF) e Guiné (G). 


\section{Vegetação do campo rupestre}

Os estudos da vegetação têm demonstrado a unidade florística do campo rupestre, onde há dominância de famílias características, como Velloziaceae, Asteraceae, Melastomataceae, Orchidaceae, Cyperaceae, Bromeliaceae, Eriocaulaceae, Poaceae e Fabaceae s.l. e alto grau de espécies endêmicas (Andrade et al. 1986; Harley \& Simmons 1986; Giulietti et al. 1987, 1996, 1997; Pinto et al. 1990; Pereira 1994; Pirani et al. 1994; Stannard 1995; Harley 1995; Vitta 1995; Guedes \& Orge 1998; Conceição \& Giulietti 2002; Conceição \& Pirani 2005; Conceição et al. 2005).

Apesar dessa unidade florística generalizada, há no campo rupestre uma notória heterogeneidade de habitats que possuem composições e estruturas diferenciadas (Pereira 1994; Vitta 1995; Giulietti et al. 1997; Conceição \& Giulietti 2002; Conceição \& Pirani 2005; Conceição et al. 2005; Conceição 2006). Em áreas extensas e planas de solos arenosos nos altos das serras há predomínio dos "campos gerais", marcado por um tapete rico em espécies de Cyperaceae, Eriocaulaceae e Poaceae. Nos locais onde as drenagens são dificultadas pelo relevo ou substrato, pode haver formação de solo húmico, sendo ocupados por espécies de Xyridaceae, Eriocaulaceae, Cyperaceae, Lentibulariaceae, Orchidaceae e Gentianaceae (Giulietti et al. 1996). Nos locais com grande extensão de superfície rochosa ocorre o habitat afloramento (Conceição \& Pirani 2005; Conceição et al. 2005), no qual muitas das espécies dominantes pertencem às famílias Velloziaceae, Clusiaceae, Orchidaceae, Bromeliaceae, Melastomataceae e Asteraceae, compondo uma fisionomia mais arbustiva do que a dos campos (Conceição \& Giulietti 2002; Conceição \& Pirani 2005). Áreas de sedimentos arenosos entre afloramentos rochosos constituem o habitat entremeio (Conceição \& Pirani 2005), bem representado por espécies de Poaceae, Cyperaceae e Fabaceae s.l., entre outras várias famílias (Conceição \& Giulietti 2002; Conceição \&
Pirani 2005; Conceição et al. 2005). Fendas no afloramento rochoso constituem o habitat vala, mais raro, onde há grande representação de eudicotiledôneas arbustivas e arbóreas de famílias como Asteraceae, Euphorbiaceae, Lamiaceae, Myrtaceae, Verbenaceae, Melastomataceae, além de criptógamas vasculares como Dryopteridaceae e Cyatheaceae (Conceição \& Pirani 2005).

\section{Amostragem}

Durante os anos de 2000 a 2002, 32 unidades de $10 \times 10 \mathrm{~m}$ foram locadas nos quatro sítios de estudo. Em cada sítio, a vegetação foi amostrada por quatro parcelas aleatórias simples de 10 x 10 m, denominadas de nãoestratificadas. Além dessas 16 parcelas, mais 16 foram estratificadas ao habitat afloramento, quatro sorteadas em cada local, totalizando 32 parcelas (ou unidades) nos quatro sítios, conforme delineamento descrito em Conceição \& Pirani (2005). A inclinação de cada unidade foi obtida a partir de triângulo retângulo medido em campo com uso de trena, nível e barbante.

As espécies de plantas vasculares em cada unidade foram determinadas e listadas (Conceição et al. 2005), sendo discernidas as riquezas em espécies criptógamas vasculares, eudicolidôneas, monocotiledôneas, assim como o número de famílias, em cada uma das 32 unidades. As partes aéreas de vegetação e de liquens foram estimadas por suas projeções verticais, resultando nas estimativas das respectivas áreas de coberturas (Westhoff \& Maarel 1978). Tais estimativas revelam aspectos da fisionomia, sendo que os liquens indicam cobertura bem próxima à superfície, enquanto a vegetação geralmente apresenta altura de 0,5 a 2,0 m (Conceição 2003). Devido à baixa representação das briófitas em relação às áreas de vegetação ou de liquens, optou-se por não quantificar sua cobertura.

\section{Análise dos dados}

Comparações entre alguns descritores de diversidade nas unidades não-estratificadas de $10 \times 10 \mathrm{~m}$ de cada local foram feitas com 
utilização da análise de variância a um critério de classificação (One way ANOVA; Callegari-Jacques 2003), calculada pelo programa Statistica 6.0. Inicialmente as variáveis foram testadas quanto à normalidade (Kolmogorov-Smirnov, $p>0,05$ ) e homocestacidade (Levene, $p>0,05$ ). No caso de rejeição da hipótese nula $(a=0,05)$ admitiuse diferença e aplicou-se o teste de Tukey para discernir as diferenças entre os locais $(\alpha=$ 0,05) (Callegari-Jacques 2003). Gráficos boxplots com médias, erros e desvios padrões também foram elaborados pelo Statistica 6.0, sendo os locais indistintos pelo teste de Tukey identificados por letras iguais.

Análises de regressão linear foram elaboradas a fim de verificar correlações do número de espécies conforme área de vegetação e inclinação das parcelas, com o número de espécies como variável dependente e testada quanto à normalidade (KolmogorovSmirnov, $p>0,05)$, sendo as demais variáveis independentes. As análises foram calculadas pelo programa Statistica 6.0. A existência de regressão na população foi testada pelo valor$P(<0,05)$ associado ao $t_{\text {calc }}$. (CallegariJacques 2003).

As espécies amostradas em apenas uma das áreas estudadas e com mais de duas ocorrências nas 32 unidades foram destacadas, assim como as espécies com sete ou oito ocorrências em ao menos uma das áreas. A classificação das 32 unidades foi realizada a partir da matriz binária com as espécies ocorrentes em três ou mais unidades, utilizando similaridade de Jaccard e a ligação dos grupos pela média nãoponderada (UPGMA).

\section{Resultados}

Foram inventariadas 202 espécies, distribuídas em 50 famílias, sendo oito criptógamas vasculares (11 espécies), 30 eudicotiledôneas (93 espécies), 11 monocotiledôneas (97 espécies) e Piperaceae (uma espécie) (lista em Conceição et al. 2005). O total de espécies na Mãe Inácia foi
78, na Fumaça 61, no Gerais da Fumaça 62 e no Guiné 124. Mais da metade das famílias possuiu de uma a duas espécies (60\%), sendo Orchidaceae a mais numerosa, com 34 espécies (Fig. 2).

Nas unidades não-estratificadas, o número de espécies $(\mathrm{S})$ e o de famílias $(\mathrm{F})$ entre os locais não variou: $F_{S(3,12)}=2,279$, $p=0,132$ e $F_{\mathrm{F}(3,12)}=0,828, p=0,503$ (Fig. $3 \mathrm{~A})$. O número de espécies eudicotiledôneas (Spp. Eudic.) e monocotiledôneas (Spp. Mono.) também foram indistintos entre os locais: $F_{\text {Spp.Eudic. }}$ ${ }_{(3,12)}=0,729, p=0,554$ e $F_{\text {Spp.Mono. (3,12) }}=$ $2,410, p=0,118$ (Fig. 3b). O número de espécies criptógamas vasculares variou significativamente entre as áreas $\left(F_{(3,12)}=\right.$ 10,364, $p=0,001$ ), sendo maior na Fumaça do que na Mãe Inácia e no Gerais da Fumaça, porém indistinto do Guiné (Fig. 3b). A área de vegetação não apresentou diferenças entre os locais: $F_{(3,12)}=0,702$, $p=0,569$ (Fig. 4a). No caso dos líquens houve diferença: $F_{(3,12)}=4,801, p=0,0202$, sendo na Mãe Inácia menor do que na Fumaça e no Gerais da Fumaça, e indistinto do Guiné (Fig. 4a).

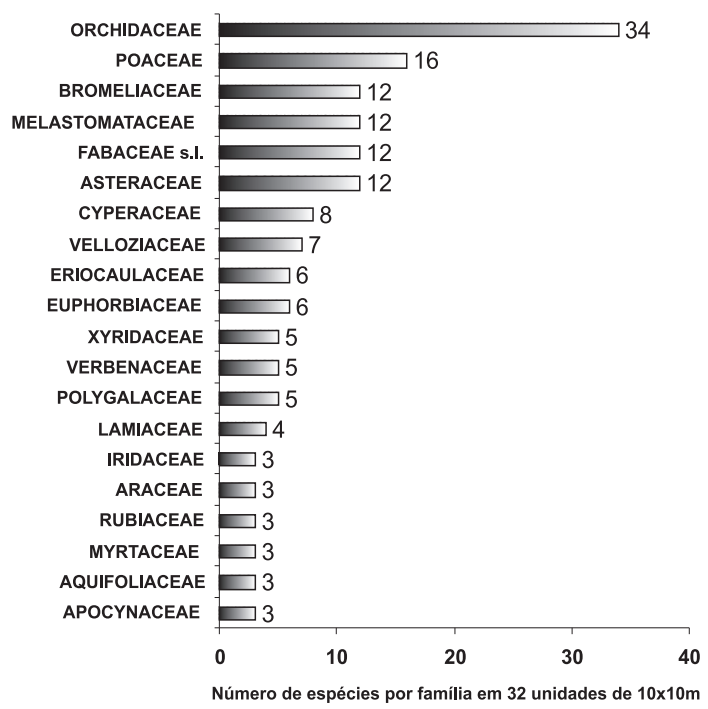

Figura 2 - Número de espécies por famílias em 32 unidades de 10 x 10 m na Mãe Inácia, Fumaça, Gerais da Fumaça e Guiné. Chapada Diamantina, Bahia, Brasil. 


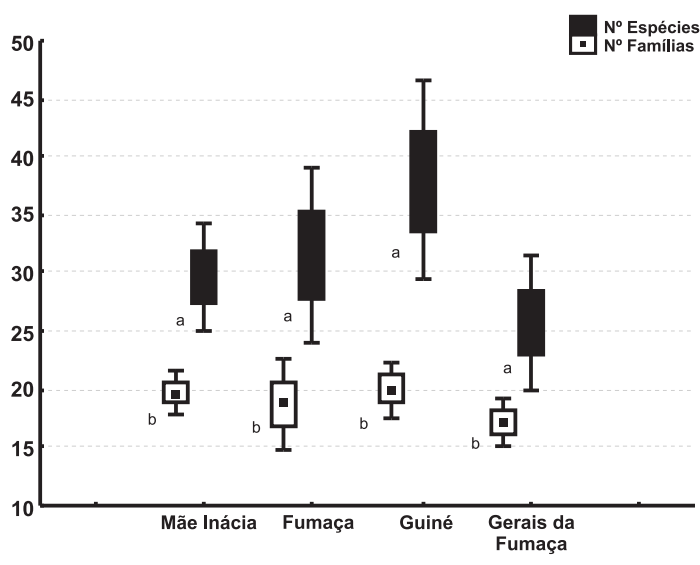

a

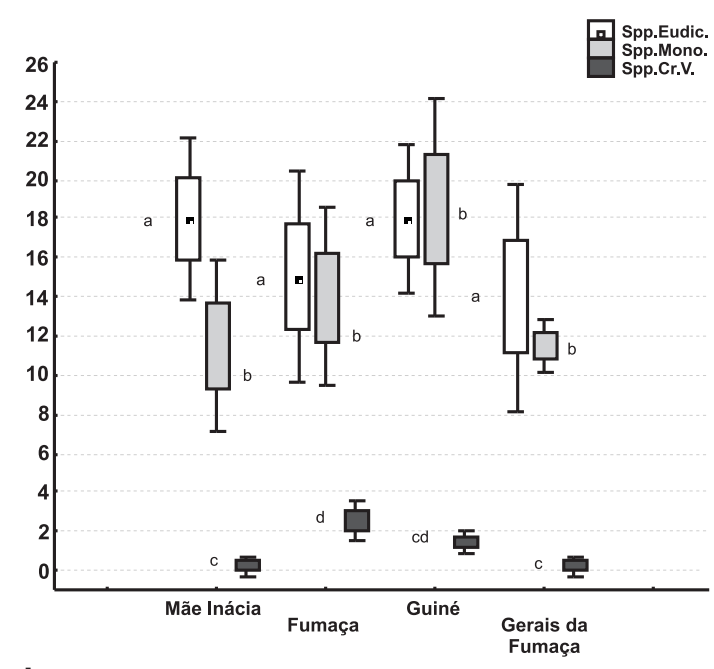

b

Local

Figura 3 - Gráfico Box-plot, com média (símbolo interno à área retangular), \pm erro padrão (área retangular) e \pm desvio padrão (barras), baseado em 16 unidades de $10 \times 10 \mathrm{~m}$ não-estratificadas nos quatro locais estudados $(n=4)$. A: número de famílias (branco) e de espécies (preto). B: espécies eudicotiledoneas (Eudic.), monocotiledôneas (Mono.) e criptógamas vasculares (Cr.V.). As letras minúsculas iguais indicam locais indistintos segundo teste de Tukey $(\alpha=0,05)$. Chapada Diamantina, Bahia, Brasil.

As retas de regressões ajustadas aos pontos relativos ao número de espécies conforme área de vegetação e inclinação revelam correlações positivas e significativas entre essas variáveis nas unidades não-estratificadas (Fig. 5 a-b)

As espécies ocorrentes em uma ou duas das 32 unidades perfizeram $46,5 \%$ das espécies. Apenas sete espécies ocorreram em mais de 65\% das 32 unidades, sendo Tibouchina pereirae Brade \& Markgr. a mais freqüente,

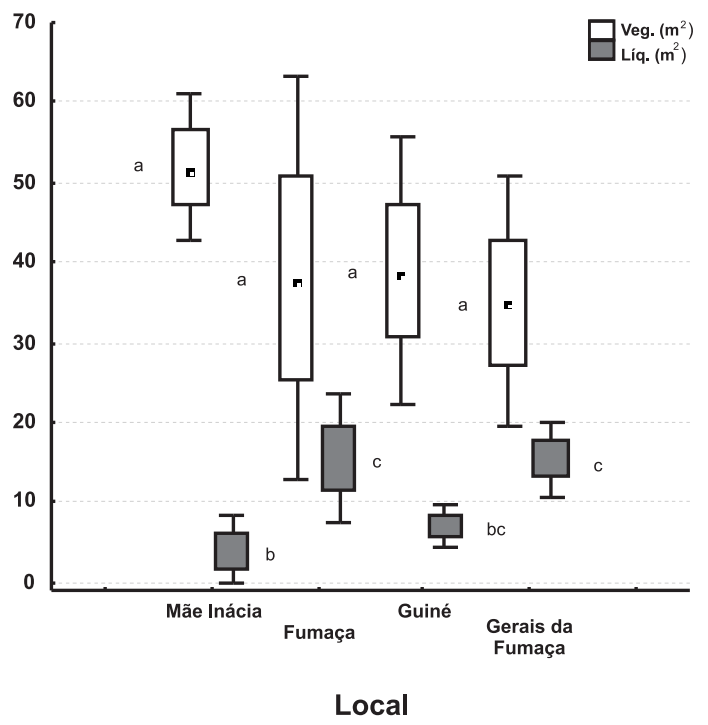

Figura 4 - Gráfico Box-plot, com média (símbolo interno à área retangular), \pm erro padrão (área retangular) e \pm desvio padrão (barras), baseado em 16 unidades de 10 x 10 m não-estratificadas nos quatro locais estudados $(\mathrm{n}=4)$. Veg: vegetação. Liq: líquen. As letras minúsculas iguais indicam locais indistintos segundo teste de Tukey $(\alpha=0,05)$. Chapada Diamantina, Bahia, Brasil.
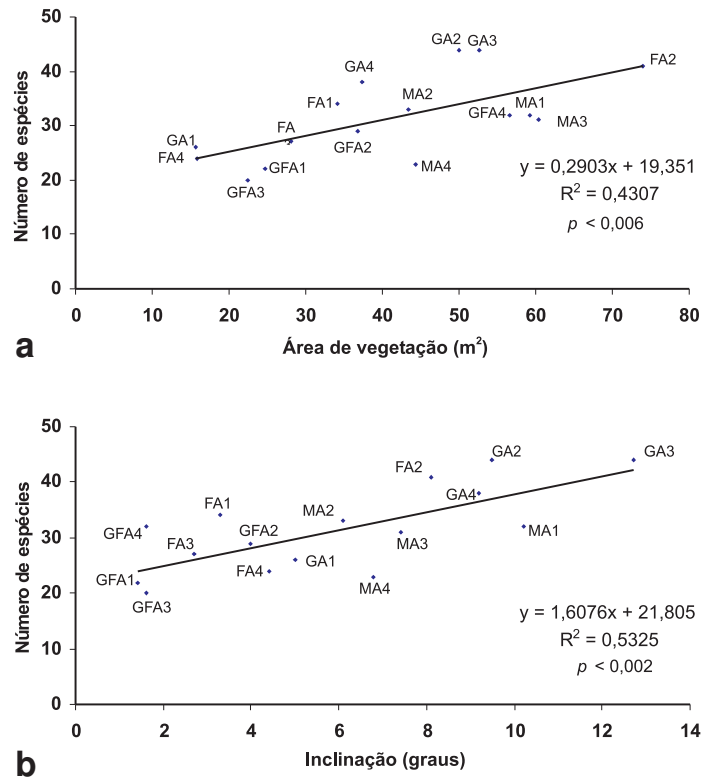

Figura 5 - Reta de regressão ajustada aos pontos relativos ao número de espécies conforme área da vegetação (A) e inclinação (B), com equação, coeficiente de determinação e significância. Os pontos referem-se às 16 unidades de 10 × 10 m não-estratificadas nos sítios Mãe Inácia (M), Fumaça (F), Gerais da Fumaça (GF) e Guiné (G). Chapada Diamantina, Bahia, Brasil. 
Tabela 1 - Espécies muito freqüentes por áreas estudadas: Mãe Inácia (M), Fumaça (F), Gerais da Fumaça (GF) e Guiné (G). Espécies em negrito foram amostradas exclusivamente em uma área da Chapada Diamantina, Bahia, Brasil.

\begin{tabular}{|c|c|c|c|c|c|c|}
\hline Áreas de estudo & Família & Espécie & M & $\mathbf{F}$ & GF & G \\
\hline \multirow[t]{2}{*}{ Nas 4} & Melastomataceae & Tibouchina pereirae Brade \& Markgr. & 8 & 7 & 8 & 7 \\
\hline & Clusiaceae & Clusia obdeltifolia Bittrich & 8 & 0 & 8 & 8 \\
\hline \multirow[t]{2}{*}{ Em 3} & Cyperaceae & Abildgaardia sp. & 4 & 8 & 8 & 7 \\
\hline & Asteraceae & Baccharis cf. salzmannii DC. & 4 & 8 & 7 & 7 \\
\hline \multirow[t]{9}{*}{ Em 2} & Orchidaceae & Epidendrum secundum Jacq. & 1 & 8 & 4 & 8 \\
\hline & Asteraceae & Lychnophora triflora (Mattf.) H.Rob. & 2 & 7 & 1 & 8 \\
\hline & Poaceae & Schizachyrium sanguineum (Retz.) Alst. & 6 & 8 & 6 & 6 \\
\hline & Asteraceae & Lasiolaena duartei R.M.King \& H.Rob. & 8 & 6 & 2 & 6 \\
\hline & Poaceae & Panicum trinii Kunth & 8 & 5 & 0 & 5 \\
\hline & Velloziaceae & Barbacenia blanchetii Goethart \& Henrard & 7 & 0 & 0 & 0 \\
\hline & Eriocaulaceae & Paepalanthus pulchellus Herzog & 0 & 8 & 5 & 4 \\
\hline & Velloziaceae & Vellozia punctulata Seub. & 0 & 8 & 0 & 0 \\
\hline & Velloziaceae & Vellozia dasypus Seub. & 6 & 7 & 0 & 1 \\
\hline \multirow[t]{8}{*}{ Em 1} & Amaryllidaceae & Hippeastrum solandriflorum (Lindl.) Herb. & 2 & 3 & 8 & 5 \\
\hline & Poaceae & Panicum cumbucana Renvoize & 1 & 1 & 8 & 5 \\
\hline & Cyperaceae & Lagenocarpus rigidus (Kunth) Nees & 0 & 0 & 8 & 6 \\
\hline & Velloziaceae & Vellozia jolyi L.B.Sm. & 0 & 0 & 8 & 8 \\
\hline & Lythraceae & Cuphea ericoides Cham. \& Schltdl. & 0 & 2 & 7 & 0 \\
\hline & Convolvulaceae & Evolvulus jacobinus Moric. & 3 & 0 & 7 & 4 \\
\hline & Bromeliaceae & Vriesea atra $\mathrm{Mez}$ & 4 & 0 & 3 & 8 \\
\hline & Orchidaceae & Sophronitis sincorana (Schltr.) Van den Berg \& M.W.Chase & 0 & 0 & 1 & 7 \\
\hline
\end{tabular}

ausente em apenas duas, representando a única espécie ocorrente em sete ou oito unidades em cada uma das quatro áreas estudadas (Tab. 1). Três espécies tiveram ocorrências elevadas em três áreas, duas espécies em duas áreas e 15 em apenas uma das áreas, sendo duas espécies de Velloziaceae amostradas exclusivamente em uma área (Tab. 1). As espécies amostradas exclusivamente em um dos locais e com mais de duas ocorrências somaram nove na Mãe Inácia, oito na Fumaça, sete no Guiné e duas no Gerais da Fumaça (Tab. 2).

A análise de agrupamento revela que as parcelas da Mãe Inácia distinguem-se das demais em $84,4 \%$ da composição, havendo formação de grupos relacionados a cada área de estudo, com similaridades superiores a $27 \%$ (Fig. 6). A Mãe Inácia constitui o grupo mais distinto, com 28,9\% de similaridade. As demais áreas são $19,9 \%$ similares entre si, com as unidades do Gerais da Fumaça e Guiné 25,7\% similares, incluindo o grupo do Guiné $(32,1 \%)$ e do Gerais da Fumaça $(32,9 \%)$. A Fumaça apresenta $32,8 \%$ de similaridade entre suas unidades. As unidades mais similares entre si são no Gerais da Fumaça (GF NE2 e GF NE4; $69,7 \%$ ) e na Fumaça (F NE1 e F NE2; 61\%).

\section{Discussão}

Estudos florísticos em outras áreas de campo rupestre na Cadeia do Espinhaço revelaram as mesmas famílias como as mais numerosas em espécies (Andrade et al. 1986; Harley \& Simmons 1986; Giulietti et al. 1987; Pereira 1994; Vitta 1995; Stannard 1995; Guedes \& Orge 1998; Conceição \& Giulietti 2002; Conceição \& Pirani 2005). Com exceção de Scrophulariaceae, as dez famílias com mais espécies em inselbergs (Barthlott \& Porembski 2000) são as mesmas deste estudo (Fig. 2). As 15 famílias com maior número de espécies em inselbergs da Guiana venezuelana também são características dos afloramentos na Chapada Diamantina (Gröger 2000), sendo que as famílias 
Tabela 2 - Espécies amostradas exclusivamente em um dos locais e com mais de duas ocorrências. Mãe Inácia (M), Fumaça (F), Gerais da Fumaça (GF) e Guiné (G). Chapada Diamantina, Bahia, Brasil.

\begin{tabular}{|c|c|c|c|c|c|}
\hline Família & Espécie & $\mathbf{M}$ & $\mathbf{F}$ & GF & $\mathbf{G}$ \\
\hline Velloziaceae & Barbacenia blanchetii Goethart \& Henrard & 7 & 0 & 0 & 0 \\
\hline Velloziaceae & Vellozia hemisphaerica Seub. & 5 & 0 & 0 & 0 \\
\hline Verbenaceae & Stachytarpheta froesii Moldenke & 5 & 0 & 0 & 0 \\
\hline Cyperaceae & Trilepis lhotzkiana Nees & 4 & 0 & 0 & 0 \\
\hline Myrtaceae & Myrcia jacobinensis Mattos & 4 & 0 & 0 & 0 \\
\hline Bromeliaceae & Hohenbergia cf. catingae Ule & 3 & 0 & 0 & 0 \\
\hline Cactaceae & Pilosocereus pachycladus F. Ritter & 3 & 0 & 0 & 0 \\
\hline Loranthaceae & Struthanthus flexicaulis Mart. & 3 & 0 & 0 & 0 \\
\hline Verbenaceae & Lippia alnifolia Schauer & 3 & 0 & 0 & 0 \\
\hline Velloziaceae & Vellozia punctulata Seub. & 0 & 8 & 0 & 0 \\
\hline Orchidaceae & Zygopetalum mackayi Hook. & 0 & 5 & 0 & 0 \\
\hline Rubiaceae & Borreria capitata (Ruiz \& Pav.) DC. & 0 & 5 & 0 & 0 \\
\hline Selaginellaceae & Selaginella marginata (Humb. \& Bonpl.) Spring & 0 & 5 & 0 & 0 \\
\hline Gesneriaceae & Paliavana tenuiflora Mansf. & 0 & 4 & 0 & 0 \\
\hline Grammitidaceae & Cochlidium serrulatum (Sw.) L.E.Bishop & 0 & 4 & 0 & 0 \\
\hline Orchidaceae & Acianthera hamosa (Barb.Rodr.) Pridgeon \& M.W.Chase & 0 & 4 & 0 & 0 \\
\hline Velloziaceae & Barbacenia sp. nov. & 0 & 3 & 0 & 0 \\
\hline Fabaceae s.l. & Calliandra viscidula Benth. & 0 & 0 & 5 & 0 \\
\hline Xyridaceae & Xyris sp. & 0 & 0 & 3 & 0 \\
\hline Bromeliaceae & Orthophytum albopictum Philcox & 0 & 0 & 0 & 6 \\
\hline Lamiaceae & Hyptis argyrophylla Harley & 0 & 0 & 0 & 6 \\
\hline Orchidaceae & Sophronitis pfiesteri (Pabst \& Senghas) Van den Berg \& M.W.Chase & 0 & 0 & 0 & 5 \\
\hline Asteraceae & Richterago discoidea (Less.) Kuntze & 0 & 0 & 0 & 3 \\
\hline Fabaceae s.l. & Calliandra mucugeana Renvoize & 0 & 0 & 0 & 3 \\
\hline Orchidaceae & Epidendrum saxatile Lindl. & 0 & 0 & 0 & 3 \\
\hline Orchidaceae & Veyretia sincorensis (Schltr.) Schltr. & 0 & 0 & 0 & 3 \\
\hline
\end{tabular}

Poaceae, Velloziaceae, Cyperaceae, Asteraceae, Melastomataceae, Orchidaceae, Bromeliaceae, Clusiaceae, Fabaceae s.l., Euphorbiaceae, Verbenaceae e Lythraceae são as mais abundantes nos afloramentos rochosos nos campos rupestres da Cadeia do Espinhaço (Pereira 1994; Meguro et al. 1994; Vitta 1995; Conceição \& Giulietti 2002; Conceição \& Pirani 2005; Conceição et al. 2005).

Esta grande afinidade florística deve-se principalmente ao substrato rochoso, que implica em severas restrições à sobrevivência das plantas (Burrows 1990; Ware 1990; Kluge \& Brulfert 2000). Estudos fitossociológicos direcionados aos afloramentos rochosos nos campos rupestres revelaram grande importância da família Velloziaceae, dada principalmente por apenas uma espécie (Pereira 1994; Conceição \& Giulietti 2002;
Conceição \& Pirani 2005) (Tabs. 1 e 2). Esta família inclui a maior porcentagem de espécies especialistas de inselbergs (Barthlott \& Porembski 2000), possuindo grande número de espécies tolerantes à dessecação (Gaff 1977; Meguro et al. 1977; Meirelles et al. 1997; Biedinger et al. 2000; Conceição 2003). A elevada cobertura de espécies de Velloziaceae e Cyperaceae tolerantes à dessecação nos afloramentos rochosos de topos de serras na Chapada Diamantina (Conceição \& Giulietti 2002; Conceição \& Pirani 2005) comprovam a importância desta estratégia para ocupação da rocha. No caso de Velloziaceae, o centro de diversidade é na Cadeia do Espinhaço (Mello-Silva 1996), sendo a ocorrência de suas espécies vinculada, essencialmente, ao substrato rochoso (Menezes 1984; Mello-Silva 1996). 


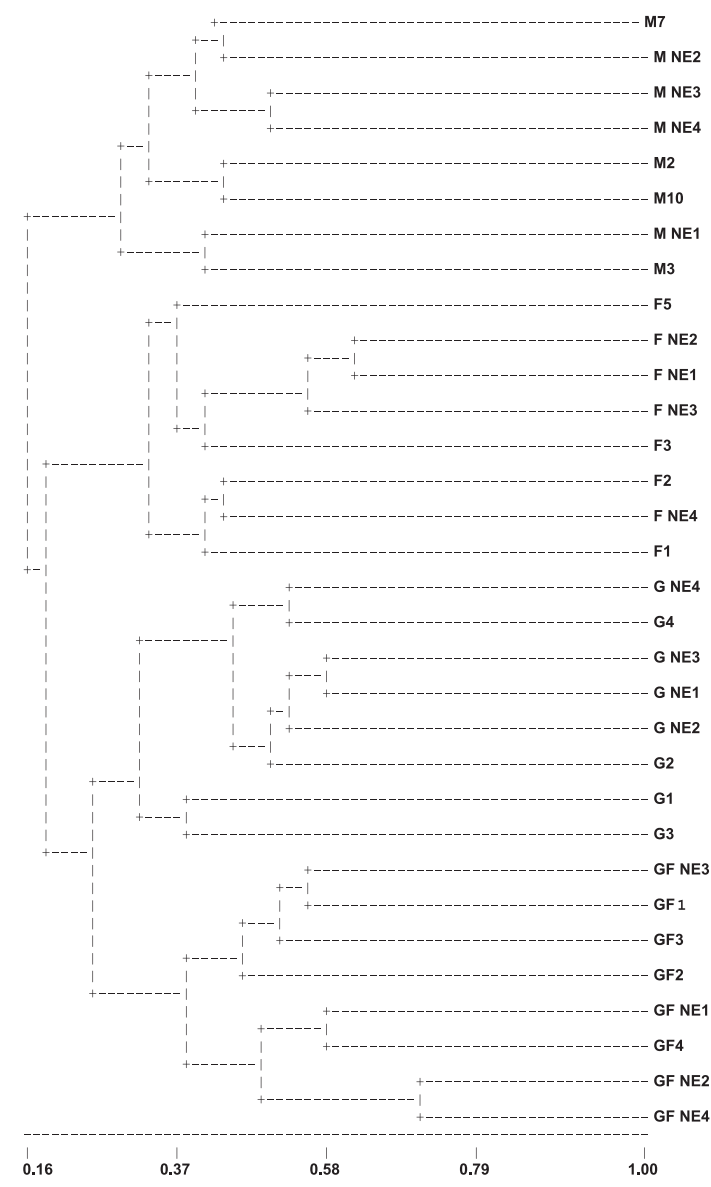

Figura 6 - Dendrograma de similaridade de Jaccard entre 32 unidades de 10 x 10 m na Mãe Inácia (M), Fumaça (F), Gerais da Fumaça (GF) e Guiné (G) na Chapada Diamantina, Bahia, Brasil. NE: parcela não-estratificada. Ligação dos grupos pela média não-ponderada (UPGMA).

O número indistinto de espécies e famílias entre os locais indica a ocorrência de um padrão similar na proporção de táxons nas áreas, independentemente das características próprias de cada local e das misturas dos habitats. Isso sugere que o ambiente rochoso de topo de morro na Chapada Diamantina seja determinante de processos similares, comportando número de espécies relativamente previsível. Tendências a um número maior ou menor sugerem processos reguladores locais. As comunidades são dominadas por quantidades distintas de determinadas espécies (Conceição \& Pirani 2005; Conceição et al. 2005), podendo ser analisado pela dinâmica de manchas, na qual manchas individuais tendem a diferir em número e/ou espécies, enquanto essa distinção no conjunto de manchas tende a ser menor (Simberloff 1976; Ribeiro \& Medina 2002; Townsend et al. 2006).

A maior riqueza de criptógamas vasculares que foi detectada na Fumaça pode estar relacionada à existência de uma cachoeira nesse local umedecendo periodicamente a vegetação com água levada pelo vento. Esse grupo de organismos possui a maioria de suas espécies em montanhas úmidas (Tryon \& Tryon 1982). Apesar da variação do número de espécies eudicotiledôneas e monocotiledôneas não ser significativa, as monocotiledôneas predominam em cobertura, principalmente devido às famílias Velloziaceae e Poaceae (Conceição \& Giulietti 2002; Conceição \& Pirani 2005). Na Mãe Inácia, a tendência ao maior número de eudicotiledôneas em relação às monocotiledôneas deve estar associada à sua elevada proporção de entremeio nas unidades não-estratificadas (Conceição \& Pirani 2005), sendo que a menor altitude a coloca mais próxima do cerrado circundante, facilitando a chegada de espécies arbustivas e arbóreas vinculadas ao cerrado, como Emmotum nitens (Benth.) Miers. Esse mesmo fator também pode estar relacionado à menor área de líquen na Mãe Inácia, colocando-a mais distante do domínio dos campos rupestres, onde há grande abundância de liquens (Conceição \& Giulietti 2002; Conceição \& Pirani 2005).

O aumento do número de espécies conforme a área da vegetação aumenta é significativo (Fig. 5), assim como na maioria dos sistemas biológicos (MacArthur \& Wilson, 1967; Rosenzweig 1995). Entretanto, no habitat entremeio estudado na Mãe Inácia e Fumaça, o número de espécies não cresce conforme o aumento da área, diferentemente do que ocorre nos habitats afloramento e vala (Conceição 2003), sugerindo que a maior extensão e conectividade do entremeio leve a uma diminuição da relação entre número de 
espécies e área, quando comparados ao afloramento e vala, mais restritos e insulares, onde há mais espécies por área.

A correlação positiva entre número de espécies e a inclinação do terreno sugere maior heterogeneidade nos locais mais inclinados, que poderiam incluir degraus na rocha, condicionando habitats distintos, maximizando a diversidade (Conceição 2003). Tal padrão pode ser esperado para a faixa de inclinação amostrada (até cerca de $13^{\circ}$ ), porém deve diferir bastante em locais muito inclinados, onde a ocupação é restrita a poucas espécies, como em encostas íngremes do Rio de Janeiro (Meirelles et al. 1999).

Ao considerar o conjunto das unidades não-estratificadas e em afloramento, o número elevado de espécies no Guiné (124) pode ser decorrente de alguns fatores, como maiores altitudes e distâncias entre as unidades, além de misturas de habitats. O número reduzido de espécies amostradas exclusivamente no Gerais da Fumaça em relação aos demais locais (Tab. 2), sugere que o menor grau de isolamento deste local facilite a chegada de espécies, como apontado pela biogeografia de ilhas (MacArthur \& Wilson 1967), conferindo maior particularidade florística aos locais com maior isolamento.

Outro fator que também pode estar associado a esse pequeno número de espécies exclusivas é o maior grau de perturbação por fogo observado no Gerais da Fumaça, onde rebrotavam rizomas de Vriesea atra queimados, além da existência de porções de superfície rochosa recentemente expostas, originadas pela lixiviação de parte do fino solo queimado. A proteção contra o fogo conferida pela ausência de combustível na rocha exposta faz com que os afloramentos sejam locais propícios ao abrigo de espécies mais sensíveis ao fogo (Biedinger et al. 2000), porém no caso do lajedo do Gerais da Fumaça, essa proteção não é tão efetiva devido à continuidade da vegetação que o circunda e se estende por quilômetros na Serra da Larguinha.
As espécies amostradas em apenas uma das áreas e ocorrentes em mais de 50\% das unidades podem ser consideradas típicas de um local, como Barbacenia blanchetii, Vellozia hemisphaerica e Stachytarpheta froesii na Mãe Inácia; Vellozia punctulata, Zygopetalum mackayi, Borreria capitata e Selaginella marginata na Fumaça; Calliandra viscidula no Gerais da Fumaça e Orthophytum albopictum, Hyptis argyrophylla e Sophronitis pfiesteri no Guiné.

A ausência de registro de uma dada espécie não significa que ela não ocorra no local, mas informa que caso ocorra, ela provavelmente não estará espacialmente bem distribuída. No caso de ocorrências repetidas na mesma área, a informação é mais elucidativa, indicando alta probabilidade de encontrar a espécie no espaço. Neste sentido, Tibouchina pereirae pode ser considerada a espécie mais típica dos locais estudados, com alta probabilidade de ser encontrada em outras regiões da Serra do Sincorá, nos habitats afloramento, entremeio e vala (Tab. 1). Dentre as espécies com elevadas ocorrências nas três áreas, Clusia obdeltifolia é a única a não ocorrer em um dos locais. Sua ausência na Fumaça é curiosa, visto que ocorre em $100 \%$ das unidades nos outros três locais, além de ser comum em várias áreas da Serra do Sincorá. Outra espécie que também se enquadra neste contexto é Vriesea atra.

De uma maneira geral, as espécies muito frequientes em mais de um local e ocorrentes em todos os locais são espécies muito comuns nas áreas de campo rupestre dominadas por afloramentos rochosos, como T. pereirae, Abildgaardia sp., Baccharis cf. salzmanii, Lychnophora triflora, Epidendrum secundum, Schizachyrium sanguineum, Lasiolaena duartei e Hippeastrum solandriflorum. As Velloziaceae Barbacenia blanchetii, Vellozia punctulata e V. jolyi são muito freqüentes onde ocorrem, ao mesmo tempo 
em que ausentes em outros. Essa é uma tendência comum observada em outras áreas, onde geralmente há domínio de uma ou duas espécies de Velloziaceae, que variam entre as áreas (Conceição \& Giulietti 2002; Conceição \& Pirani 2005). A grande abundância da família Velloziaceae nos afloramentos rochosos estudados pode ser consequiência de um ajuste de suas espécies com a irregularidade do clima. No período seco elas tendem a se desidratarem, tornando ao estado hidratado com as chuvas (Conceição 2003). Além dessa habilidade, elas possuem florações sincronizadas com as chuvas (Ayensu 1973; Conceição 2003).

O dendrograma de similaridade demonstra a grande variação na composição de espécies entre as áreas estudadas, fator relacionado à elevada diversidade de espécies (Whittaker 1977), particularmente importante em sistemas insulares, que aliam isolamento, fatores históricos e estocásticos e particularidades ambientais (Whittaker et al. 2001). O fato das unidades da Mãe Inácia constituírem o grupo mais distinto pode estar relacionado ao seu maior isolamento na paisagem.

$\mathrm{O}$ fato do Gerais da Fumaça ser mais similar com o Guiné ao invés de com a Fumaça, indica complexidade de fatores relacionados à composição de espécies. Esperava-se que o Gerais da Fumaça e a Fumaça seriam os locais mais similares, já que são bem mais próximos entre si (Fig. 1), onde os fatores edáficos e climáticos seriam mais parecidos, além da maior facilidade de dispersão das espécies para áreas próximas. As elevadas similaridades entre unidades do Gerais da Fumaça podem ser relacionadas à tendência ao menor número de espécies nesse local, aumentando a probabilidade de coocorrências de espécies.

\section{Conclusão}

A existência de padrões de diversidade similares em locais distintos, relacionados aos números de espécies e famílias, assim como número de espécies eudicotiledôneas e monocotiledôneas, além das áreas de vegetação, sugerem que os fatores vinculados à vegetação dos afloramentos rochosos determinem processos similares, mesmo que em áreas distintas. Entretanto, diferenças quanto aos números de criptógamas vasculares e áreas de liquens são provavelmente relacionadas às peculiaridades locais, que aliadas à grande variação na composição de espécies entre os locais (diversidade regional ou epsilon, sensu Whittaker 1977), constituem fatores determinantes à elevada diversidade desse tipo de vegetação.

\section{Agradecimentos}

À Fapesp pela bolsa de doutorado, ao Ibama pela permissão de coleta no Parque Nacional e às sugestões de J. A. N. Conceição.

\section{REFERÊNCIAS BIBLIOGRÁFICAS}

Alves, R. J. V. \& Kolbek, J. 1993. Penumbral rock communities in campo rupestre sites in Brazil. Journal of Vegetation Science 4: 357-366.

Andrade, P. M.; Gontijo, T. A. \& Grandi, T. S. M. 1986. Composição florística e aspectos estruturais de uma área de "campo rupestre" do Morro do Chapéu, Nova Lima, Minas Gerais. Revista Brasileira de Botânica 9: 13-21.

Ayensu, E. S. 1973. Biological and morphological aspects of the Velloziaceae. Biotropica 5(3): 135-149.

Barthlott, W. \& Porembski, S. 2000. Vascular plants on inselbergs: systematic overview. In: Porembski, S. \& Barthlott, W. Inselbergs. Ecological Studies. Vol. 146. Springer-Verlag, Heidelberg. Pp. 103-116. 
Biedinger, N.; Porembski, S. \& Barthlott, W. 2000. Vascular plants on inselbergs: vegetative and reproductive strategies. In: Porembski, S. \& Barthlott, W. Inselbergs. Ecological Studies. Vol. 146. SpringerVerlag, Heidelberg. Pp. 117-142.

Burrows, C. J. 1990. Processes of vegetation change. Urwin Hyman, London, 551p.

Callegari-Jacques, S. M. 2003. Bioestatística: princípios e aplicações. Artmed, Porto Alegre, 255p.

Conceição, A. A. 2000. Alerta para a conservação da biota na Chapada Diamantina. Revista Ciência Hoje 27(159): 54-56.

2003. Ecologia da vegetação em afloramentos rochosos na Chapada Diamantina, Bahia, Brasil. Tese de Doutorado. Universidade de São Paulo, São Paulo.

2006. Plant ecology in 'campos rupestres' of the Chapada Diamantina, Bahia. In: Queiroz, L. P.; Rapini, A. \& Giulietti, A. M. Towards greater knowledge of the brazilian semi-arid biodiversity. Ministério da Ciência e Tecnologia, Brasília. Pp. 63-67.

\& Giulietti, A. M. 2002. Composição florística e aspectos estruturais de campo rupestre em dois platôs do Morro do Pai Inácio, Chapada Diamantina, Bahia, Brasil. Hoehnea 29(1): 37-48.

\& Pirani, J. R. 2005. Delimitação de habitats em campos rupestres na Chapada Diamantina: substratos, composição florística e aspectos estruturais. Boletim de Botânica da Universidade de São Paulo 23(1): 85-111.

; Rapini, A.; Pirani, J. R.; Giulietti, A. M; Harley, R. M.; Silva, T. R.; Santos, A. K.; Correia, C.; Andrade, I. M.; Costa, J. A. S.; Souza, L. R. S.; Andrade, M. J. G.; Funch, R. R.; Freitas, T. A.; Freitas, A. M. M. \& Oliveira, A. A. 2005. Campos Rupestres. In: Juncá, F. A.; Funch, L. \& Rocha, W. Biodiversidade e Conservação da Chapada Diamantina. Ministério do Meio Ambiente, Brasília. Pp. 153-180.

Gaff, D. F. 1977. Desiccation tolerant vascular plants of Southern Africa. Oecologia 31: 95-109.

Giulietti, A. M.; Menezes, N. L.; Pirani, J. R.; Meguro, M. \& Wanderley, M. G. L. 1987. Flora da Serra do Cipó, Minas Gerais: caracterização e lista das espécies. Boletim de Botânica da Universidade de São Paulo 9: 1-151.

\& Pirani, J. R. 1988. Paterns of geographic distribution of some plant species from the Espinhaço Range, Minas Gerais and Bahia, Brazil. In: Vanzolini, P. E. \& Heyer, W. R. Proceedings of a workshop on neotropical distribution patterns. Academia Brasileira de Ciências, Rio de Janeiro. Pp. 39-69.

; Pirani, J. R. \& Harley, R. M. 1997. Espinhaço Range Region, Eastern Brazil. In: Davis, S. D.; Heywood, V. H.; Herrera-Macbryde, O.; Villa-Lobos, J. \& Hamilton, A. C. Centres of plant diversity. A guide and strategy for their conservation. Vol. 3. The Americas. IUCN Publication Unity, Cambridge. Pp. 397-404.

; Queiroz, L. P. \& Harley, R. M. 1996. Vegetação e flora da Chapada Diamantina, Bahia. Anais $4^{\mathrm{a}}$ reunião especial da SBPC, Feira de Santana. Pp. 144-156.

Gröger, A. 2000. Flora and vegetation of inselbergs of venezuelan guayana. In: Porembski, S. \& Barthlott, W. Inselbergs. Ecological Studies. Vol. 146. SpringerVerlag, Heidelberg. Pp. 291-314.

Guedes, M. L. S. \& Orge, M. D. R. 1998. Check-list das espécies vasculares do Morro do Pai Inácio (Palmeiras) e da Serra da Chapadinha (Lençóis), Chapada Diamantina, Bahia, Brasil. Universidade Federal da Bahia, Salvador, 69p. 
Harley, R. M. 1995. Introduction. In: Stannard, B. L. Flora of the Pico das Almas, Chapada Diamantina, Brazil. Royal Botanic Gardens, Kew. Pp. 1-42.

\& Simmons, N. A. 1986. Florula of Mucugê, Chapada Diamantina - Bahia, Brazil. Royal Botanic Gardens, Kew, 227p.

Kluge, M. \& Brulfert, J. 2000. Ecophysiology of vascular plants on inselbergs. In: Porembski, S. \& Barthlott, W. Inselbergs. Ecological Studies. Vol. 146. SpringerVerlag, Heidelberg. Pp. 143-174.

MacArthur, R. H. \& Wilson, E. O. 1967. The theory of island biogeography. Princeton University Press, Princeton, 203p.

Meguro, M.; Joly, C. A. \& Bittencourt, M. M. 1977. Stress hídrico e alguns aspectos do comportamento fisiológico em Xerophyta plicata Spreng. - Velloziaceae. Boletim de Botânica da Universidade de São Paulo 5: 27-42.

; Pirani, J. R.; Giulietti, A. M. \& MelloSilva, R. 1994. Phytophysiognomy and composition of the vegetation of Serra do Ambrósio, Minas Gerais, Brazil. Revista Brasileira de Botânica 17(2): 149-166.

Meirelles, S. T.; Mattos, E. A. \& Silva, A. C. 1997. Potential desiccation tolerant vascular plants from Southeastern Brazil. Polish Journal of Environmental Studies 6(4): 17-21.

; Pivello, V. R. \& Joly, C. A. 1999. The vegetation of granite rock outcrops in Rio de Janeiro, Brazil, and the need for its protection. Environmental Conservation 26(1): 10-20.

Mello-Silva, R. 1996. Revisão das Vellozia tubifloras (Vellozia sect. Radia) e caracteres para o aprimoramento da filogenia de Velloziaceae. Tese de Doutorado. Universidade de São Paulo, São Paulo.

Menezes, N. L. 1984. Características anatômicas e a filogenia, na família Velloziaceae. Tese Livre de Docência, Universidade de São Paulo, São Paulo.
Moreira, A. A. N. \& Camelier, C. 1977. Relevo. In: Geografia do Brasil: Região Nordeste. Vol.2. Fundação Instituto Brasileiro de Geografia e Estatística, Rio de Janeiro, 454p.

Nimer, N. 1989. Climatologia do Brasil. $2^{\mathrm{a}}$ ed. IBGE, Rio de Janeiro, 421p.

Pereira, M. C. 1994. Estrutura das comunidades vegetais de afloramentos rochosos dos campos rupestres do Parque Nacional da Serra do Cipó, MG. Dissertação de Mestrado. Universidade Federal de Minas Gerais, Belo Horizonte.

Pinto, G. C.; Bautista, H. P. \& Lima, J. C. 1990. A Chapada Diamantina, sua fitofisionomia e peculiaridades florísticas. In: Anais do $35^{\circ}$ Congresso Nacional de Botânica. Sociedade Botânica do Brasil, Brasília. Pp. 256-295.

Pirani, J. R.; Giulietti, A. M.; Mello-Silva, R. \& Meguro, M. 1994. Checklist and patterns of geographic distribution of the vegetation of Serra do Ambrósio, Minas Gerais, Brazil. Revista Brasileira de Botânica 17(2): 133-147.

Prance, G. T. 1994. The use of phytogeographic data for conservation planning. In: Forey, P. I.; Humphries, C. J. \& Vane-Wright, R. I. Systematics and Conservation Evaluation. Systematics Association Special. Vol. 50. Clarendon Press, Oxford. Pp. 145-163.

Ribeiro, K. T. \& Medina, B. M. O. 2002. Estrutura, dinâmica e biogeografia das ilhas de vegetação sobre rocha do Planalto do Itatiaia, RJ. Boletim do Parque Nacional do Itatiaia 10: 11-82.

Rosenzweig, M. L. 1995. Species diversity in space and time. Cambridge University Press, Cambridge, 436p.

Simberloff, D. 1976. Species turnover and equilibrium island biogeography. Science 194: 572-578.

Stannard, B. L. 1995. Flora of the Pico das Almas, Chapada Diamantina, Brazil. Royal Botanic Gardens, Kew, 853p. 
Torquato, J. R. \& Fogaça, A. C. C. 1981. Correlação entre o supergrupo Espinhaço no Brasil, o grupo Chela em Angola e as formações Nasib e Khoabendus da Namíbia. In: Anais do simpósio sobre o Craton do São Francisco e suas faixas marginais. Sociedade Brasileira de Geologia, Núcleo da Bahia, Coordenação da Produção Mineral. Pp.87-99.

Townsend, C. R.; Begon, M \& Harper, J. L. 2006. Fundamentos em Ecologia. Artmed, Porto Alegre, 592p.

Tryon, R. M. \& Tryon, A. F. 1982. Ferns and allied plants with special reference to Tropical America. Springer-Verlag, New York, 857p.

Vitta, F. A. 1995. Composição florística e ecologia de comunidades campestres na Serra do Cipó, Minas Gerais. Dissertação de Mestrado. Universidade de São Paulo, São Paulo.

Ware, S. 1990. Adaptation to substrate-and lack of it-in rock outcrop plants: Sedum and Arenaria. American Journal of Botany 77(8): 1095-1100.

Westhoff, V. \& Maarel, E. van der. 1978. The Braun-Branquet Approach. In: Whittaker, R. H. Classification of plant communities. Dr W. Junk, Boston. Pp. 287-399.

Whittaker, R. H. 1977. Evolution of species diversity in land communities. In: Hecht, M. K.; Steere, W. C. \& Wallace, B. Evolutionary biology. Vol. 10. Plenum Press, New York. Pp. 1-67.

Whittaker, R. J.; Willis, K.J. \& Field, R. 2001. Scale and species richness: towards a general hierarchical theory of species diversity. Journal of Biogeography 28: 453-470.

Zappi, D. C.; Lucas, E.; Stannard, B. L.; Niclughadha, E.; Pirani, J. R.; Queiroz, L. P.; Atkins, S.; Hind, D. J. N.; Giulietti, A. M.; Harley, R. M. \& Carvalho, A. M. 2003. Lista das plantas vasculares de Catolés, Chapada Diamantina, Bahia, Brasil. Boletim de Botânica da Universidade de São Paulo 21(2): 345-398. 